hep-th/0010218

HU Berlin-EP-00/43

\title{
Some Computations of Partition Functions and Tachyon Potentials in Background Independent Off-Shell String Theory
}

\author{
Oleg Andreev*† \\ Humboldt-Universität zu Berlin, Institut für Physik \\ Invalidenstraße 110, D-10115 Berlin, Germany
}

\begin{abstract}
We discuss what information can be safely extracted from background independent off-shell string theory. The major obstacle in doing so is that renormalization conditions of the underlying worldsheet theories are not exactly known. To get some insight, we first consider the tachyon and gauge field backgrounds and carry out computations in different renormalization schemes for both, bosonic string and superstring. Next, we use a principle of universality (renormalization scheme independence) to somehow compensate the missing of the renormalization conditions and get information we are looking for. It turns out that some asymptotics which are responsible for the potentials only obey the principle of universality.
\end{abstract}

PACS : 11.25.Sq

Keywords: background independent open string field theory

\section{$1 \quad$ Introduction}

The background independent open string theory (BIOST) 11, 2, 3, 4 turns out to be a powerful tool to compute the exact tree level tachyon potentials [5, 6, 7] which amusingly coincide with the potentials found within toy models based on the exactly solvable Schroedinger problem [8, 9]. Such a theory essentially uses two-dimensional world-sheet theories on the disk which are free field theories inside the disk but includes non-trivial interactions at the boundary $\mathrm{W}$.

From the beginning, it was emphasized in [1] that the construction of BIOST is rather formal as it ignores ultraviolet divergences associated with unrenormalizable world-sheet interactions. Moreover, it is not known precisely what the renormalization conditions for world-sheet theories are. Thus it is not clear what information can been safely extracted from this approach; clarifying this will be the goal of the present paper. To do so, we restrict ourself to renormalizable world-sheet interactions which correspond to the tachyon and gauge field backgrounds that allows us to use the whole machinery of the renormalization group ( $R G)$. As to the renormalization conditions, our main requirement which will replace their missing will be universality. In other words, we believe in structures in BIOST actions which are independent of a renormalization scheme used for world-sheet theories.

Our conventions and some features of the Witten background independent open string theory that are relevant to our discussion are the following:

\footnotetext{
*e-mail: andreev@physik.hu-berlin.de

${ }^{\dagger}$ Permanent address: Landau Institute for Theoretical Physics, Moscow, Russia

${ }^{1}$ For earlier discussions of world-sheet theories see, e.g., [10] and references therein.
} 
(i) In the bosonic case, the world-sheet action is given by

$$
S=S_{0}+S_{b}
$$

where the bulk action $S_{0}$ is

$$
S_{0}=\frac{1}{4 \pi} \int_{D} d^{2} z\left(\partial X^{\mathrm{T}} \bar{\partial} X+4 b \bar{\partial} c+\text { c.c. }\right)
$$

while the boundary action $S_{b}$ is

$$
S_{b}=\oint_{\partial D} d \theta V=a+\frac{1}{8 \pi} \oint_{\partial D} d \theta\left(X^{\mathrm{T}} t X+i \dot{X}^{\mathrm{T}} f X\right)
$$

Our notations are usual: $D$ is the disk (string world-sheet) whose coordinates are $(z, \bar{z})$; $\partial D$ is its boundary parameterized by $\theta ; X^{i}$, s are the matter fields that map the world-sheet into space-time with metric $\delta_{i j} ; b$ and $c$ are the anti-ghost and ghost fields; $a, t_{i j}, f_{i j}$ are some parameters (coupling constants).

In fact, the action as it is written in above is the ordinary bosonic open string $\sigma$-model action with special profiles for the background tachyon and gauge fields.

(ii) The partition function of the mater sector

$$
Z(a, t, f)=\int \mathcal{D} X \exp (-S)
$$

is simply found through the partial differential equations (see [1, 2] for details). In our case, the result of formal manipulations can be written as

$$
\frac{\partial \ln Z}{\partial t}=-\frac{1}{4}\left\langle X^{\mathrm{T}}(\theta) X(\theta)\right\rangle \quad, \quad \frac{\partial \ln Z}{\partial f}=-\frac{i}{4}\left\langle\dot{X}^{\mathrm{T}}(\theta) X(\theta)\right\rangle
$$

with the exact Green's function $\left\langle X(z) X\left(z^{\prime}\right)\right\rangle$ evaluated at boundary points [2]

$$
\left\langle X^{\mathrm{T}}(\theta) X\left(\theta^{\prime}\right)\right\rangle=\frac{2}{t}+2 \sum_{k=1}^{\infty} \frac{\mathrm{e}^{i k\left(\theta-\theta^{\prime}\right)}}{k+t+k f}+\frac{\mathrm{e}^{-i k\left(\theta-\theta^{\prime}\right)}}{k+t-k f}
$$

The equations (1.5) can be solved by

$$
Z(a, t, f)=(\operatorname{det} t)^{-\frac{1}{2}} \mathrm{e}^{-a} \prod_{k=1}^{\infty} \operatorname{det}\left(1+f+k^{-1} t\right)^{-1}
$$

Before continuing our discussion, let us make a couple of general comments: (1) by the differential equations $Z$ is defined up to an overall normalization constant $Z(0)$ which we omit. Moreover, $Z(0)$ may be in general divergent. (2) The expression (1.7) is purely formal as the infinite product is divergent. We postpone discussion of this complication until section 2 .

(iii) The background independent open string theory action proposed by Witten based on the Batalin-Vilkovisky formalism is defined as [1]

$$
d S_{w}=\frac{1}{2} \oint_{\partial D} \oint_{\partial D} d \theta d \theta^{\prime}\left\langle d O(\theta)\{Q, O\}\left(\theta^{\prime}\right)\right\rangle \quad, \quad\langle\ldots\rangle=\int \mathcal{D} X \mathcal{D} c \mathcal{D} b \exp (-S)
$$

\footnotetext{
${ }^{2}$ For the sake of simplicity, we use the matrix notations here and below.
} 
where $O(\theta)=c V(\theta) ; c$ is a tangential component of the ghost field at the boundary. $Q$ means the BRST operator coming from the bulk world-sheet action i.e., in other words, from closed string and as a consequence it does not depend on the couplings. Eq.(1.8) presents a set of partial differential equations which after integration results in the boundary string field theory action. According to general arguments 迎, 目, the solution is given by

$$
S_{w}=\left(1+V^{i} \frac{\partial}{\partial \mathrm{x}^{i}}\right) Z
$$

where $V^{i}$ is a vector field on the space of couplings $\mathrm{x}^{i}$.

At this point, let us mention that there is a very similar relation between $S$ and $Z$ [便 namely,

$$
S_{s}=\left(1+\beta^{i} \frac{\partial}{\partial \mathrm{x}^{i}}\right) Z
$$

where $\beta^{i}$ is the RG $\beta$-function. It was checked within the perturbation theory up to the second order and then it was suggested that it is exact in all orders. In fact, (1.10) assumes a different definition of the BRST operator because it now depends on the couplings (see [4] for further details). Thus one has to consider this as the second definition of the BIOST action. So far, it is unknown which action is better. Since the normalization conditions within the world-sheet renormalization group are missing it makes no sense to compare these actions. What we can compare is some universal structures and we will see in section 3 that these structures turned out to be the same in the both cases, so from our point of view these actions are equivalent.

Finally, let us note that both actions are defined modulo the normalization which can be fixed by comparing the on-shell three tachyon amplitude computed from these actions with the amplitude computed via the cubic open string field theory (see [11]).

(iv) In the fermionic case we follow the NSR formalism, so the world-sheet action is given by

$$
\hat{S}=\hat{S}_{0}+\hat{S}_{b}
$$

where the bulk action $\hat{S}_{0}$ is

$$
\hat{S}_{0}=\frac{1}{4 \pi} \int_{D} d^{2} z\left(\partial X^{\mathrm{T}} \bar{\partial} X+\Psi^{\mathrm{T}} \bar{\partial} \Psi+4 b \bar{\partial} c+4 \beta \bar{\partial} \gamma+\text { c.c. }\right)
$$

while the boundary action $\hat{S}_{b}$ is f

$$
\hat{S}_{b}=\oint_{\partial D} d \theta \hat{V}=a+\frac{1}{8 \pi} \oint_{\partial D} d \theta\left(X^{\mathrm{T}} t X+\psi^{\mathrm{T}} t \partial_{\theta}^{-1} \psi+i \dot{X}^{\mathrm{T}} f X-i \psi^{\mathrm{T}} f \psi\right),
$$

with $\left.\Psi\right|_{\partial D}=\left.\bar{\Psi}\right|_{\partial D}=\psi$.

The boundary action (1.13) is a natural supersymmetrization of the bosonic boundary action (1.3). The supersymmetry transformation acts on the fields in the standard way

$$
\delta X=\psi \varepsilon \quad, \quad \delta \psi=-\dot{X} \varepsilon
$$

\footnotetext{
${ }^{3}$ We do not write down $V$ explicitly leaving its discussion to section 3.

${ }^{4}$ We got such a form by using one-dimensional SUSY and the corresponding bosonic action. One can also start from the Witten vertex operator 12] and then integrating away the auxiliary fields get the same result for a linear tachyon profile $T(X)=u X$ (see [7] for details). Note that it gives $t=u^{2}$.
} 
As in the bosonic case the partition function of the matter sector

$$
\hat{Z}(a, t, f)=\int \mathcal{D} X \mathcal{D} \Psi \mathcal{D} \bar{\Psi} \exp (-\hat{S})
$$

can be also found through the partial differential equations. This time they are given by

$$
\begin{aligned}
& \frac{\partial \ln \hat{Z}}{\partial t}=-\frac{1}{4}\left\langle X^{\mathrm{T}}(\theta) X(\theta)\right\rangle-\frac{1}{4}\left\langle\psi^{\mathrm{T}}(\theta) \partial_{\theta}^{-1} \psi(\theta)\right\rangle, \\
& \frac{\partial \ln \hat{Z}}{\partial f}=-\frac{i}{4}\left\langle\dot{X}^{\mathrm{T}}(\theta) X(\theta)\right\rangle+\frac{i}{4}\left\langle\psi^{\mathrm{T}}(\theta) \psi(\theta)\right\rangle,
\end{aligned}
$$

with $\left\langle X^{\mathrm{T}} X\right\rangle$ and $\left\langle\psi^{\mathrm{T}} \psi\right\rangle$ being the exact Green's functions evaluated at boundary points. The first has been already given by (1.6). As to the second, it is simply

$$
\left\langle\psi^{\mathrm{T}}(\theta) \psi\left(\theta^{\prime}\right)\right\rangle=2 i \sum_{r=\frac{1}{2}}^{\infty} \frac{r \mathrm{e}^{i r\left(\theta-\theta^{\prime}\right)}}{r+t+r f}-\frac{r \mathrm{e}^{-i r\left(\theta-\theta^{\prime}\right)}}{r+t-r f} .
$$

Since we consider the Neveu-Schwarz sector $r$ is a half integer number.

Formally, the equations (1.16)-(1.17) can be solved by

$$
\hat{Z}(a, t, f)=(\operatorname{det} t)^{-\frac{1}{2}} \mathrm{e}^{-a} \prod_{k=1}^{\infty} \operatorname{det}\left(1+f+k^{-1} t\right)^{-1} \prod_{r=\frac{1}{2}}^{\infty} \operatorname{det}\left(1+f+r^{-1} t\right)
$$

Once more, before continuing our discussion, let us pause to make a couple of general comments: (1) by the differential equations $\hat{Z}$ is defined up to an overall additive normalization constant $\hat{Z}(0)$ we omit. (2) The expression (1.19) is purely formal as the infinite products are divergent. In general, it is natural to expects that SUSY may help us with fixing the problem i.e., the divergences cancel each other. The point however is whether this eliminates all divergencies or only some of them. We do know one thing about one-dimensional SUSY [13]. Whatever it does, it can not completely eliminate logarithmic divergences. In section 2, we will see whether it happens in the problem at hand.

(v) Unfortunately, in the fermionic case the notion of the BIOST action as it is given by (1.8) is still missing. Following 沟, it seems reasonable to try

$$
\hat{S}_{s}=\left(1+\beta^{i} \frac{\partial}{\partial \mathrm{x}^{i}}\right) \hat{Z}
$$

We need to make a couple of remarks here. First, every time the actions are defined as functions of the renormalized couplings. Second, a proposal (1.20) differs from another recent proposal of [0] namely,

$$
\hat{S}=\hat{Z}
$$

This definition inspired by [13] coincides with (1.20) only at the RG fixed points. A natural question to pose is whether these definitions lead to the same result. Again, since the renormalization conditions are missing it makes not a lot of sense to compare them. What we can compare is some universal structures. We postpone discussion of this issue until section 3.

The outline of the paper is as follows. We start in section 2 by evaluating the partition functions in different renormalization schemes to look for universal structures in them. We did so for the tachyon and gauge field backgrounds in the both, bosonic and fermionic, cases. Thus, this can be also considered 
as a generalization of [5, 6, 7] to the case of non-zero gauge field 9. Section 3 will present the application of our results to the BIOST actions. We show that from our point of view (universality) there is no difference between two definitions of the action in the bosonic case. Both of them result in the same expression which coincides with the result of [5, 6] for vanishing gauge field. We also probe our proposal (1.20) for superstring and show that it gives the same potential as in [7, 9]. Section 4 will present our conclusions and remarks.

\section{Evaluation of the Partition Function}

\subsection{The bosonic string}

We now turn to the problem of the evaluation of the partition function. Actually, the expression for $Z$ as we've already emphasized is purely formal since it is divergent due to the infinite product. To make sense of the partition function, one has to introduce a regularization procedure. In papers [1], 2n, it was done by introducing the normal ordering prescription or short-distance cut-off. In general, dealing with functional determinants means that it is more advantageous to use the so-called $\zeta$-function regularization which is based on the Riemann $\zeta$-function 6. Note that this was the case in 15 where the partition function without the tachyon field was found. So, let us begin with the $\zeta$-function regularization scheme.

The expression (1.7) can be rewritten as

$$
Z(a, t, f)=(\operatorname{det} t)^{-\frac{1}{2}} \exp \left(-a-\zeta(0) \operatorname{tr} \ln (1+f)-\sum_{k=1}^{\infty} \operatorname{tr} \ln \left(1+k^{-1} \frac{1}{1+f} t\right)\right) .
$$

Expanding $\ln \left(1+k^{-1} \frac{1}{1+f} t\right)$ in a power series and collecting terms, one finds

$$
Z(a, t, f)=(\operatorname{det} t)^{-\frac{1}{2}} \exp \left(-a-\zeta(0) \operatorname{tr} \ln (1+f)-\zeta(1) \operatorname{tr}\left(\frac{1}{1+f} t\right)+\sum_{n=2}^{\infty} \frac{(-)^{n}}{n} \zeta(n) \operatorname{tr}\left(\frac{1}{1+f} t\right)^{n}\right) .
$$

Using also $\zeta(0)=-\frac{1}{2}$, this becomes

$$
Z(a, t, f)=(\operatorname{det} u)^{-\frac{1}{2}} \exp \left(-a-\zeta(1) \operatorname{tr} u+\sum_{n=2}^{\infty} \frac{(-)^{n}}{n} \zeta(n) \operatorname{tr} u^{n}\right),
$$

with

$$
u=\frac{1}{1+f} t
$$

If we closely look at the result (2.3), it is clear that $Z$ is divergent as anticipated 7 and, moreover, the dependence on $(t, f)$ enters only through a special combination $\frac{1}{1+f} t$. We renormalize the couplings via the minimal subtraction

$$
a=a^{\prime}-\zeta(1) \operatorname{tr} u^{\prime} \quad, \quad u=u^{\prime} \quad, \quad \text { with } \quad t=t^{\prime} \quad, \quad f=f^{\prime}
$$

\footnotetext{
${ }^{5} \mathrm{~A}$ recent discussion that has overlap with what we describe in the case of bosonic string is due to [14]. It follows the original paper [1], however, without discussing ambiguity of the results. Note that earlier discussion is due to [2].

${ }^{6}$ The $\zeta$-function is defined by $\zeta(z, q)=\sum_{n=0}^{\infty} \frac{1}{(n+q)^{z}}$, with $\zeta(z)=\zeta(z, 1)$.

${ }^{7}$ Note that $\zeta(1)=\infty$. Moreover, $z=1$ is the only singular point of $\zeta(z)$.
} 
where $(a, t, f)$ are the bare couplings and $\left(a^{\prime}, t^{\prime}, f^{\prime}\right)$ are the renormalized ones.

Next we use the renormalization (2.5), and write the renormalized partition function, $Z^{\prime}\left(a^{\prime}, t^{\prime}, f^{\prime}\right) \equiv$ $Z(a, t, f)$, in terms of the new variables $\left(a^{\prime}, u^{\prime}\right)$

$$
Z^{\prime}\left(a^{\prime}, u^{\prime}\right)=\left(\operatorname{det} u^{\prime}\right)^{-\frac{1}{2}} \exp \left(-a^{\prime}+\sum_{n=2}^{\infty} \frac{(-)^{n}}{n} \zeta(n) \operatorname{tr}\left(u^{\prime}\right)^{n}\right) .
$$

To simplify further, we will use the following matrix identity

$$
\ln \Gamma(1+M)=\sum_{n=2}^{\infty} \frac{(-)^{n}}{n} \zeta(n) M^{n}-\gamma M,
$$

where $M$ is a matrix; $\gamma$ denotes the Euler's constant. Note that it is simply a matrix generalization of the identity for the logarithm of the $\gamma$-function [16]. Thus we end up with the renormalized partition function

$$
Z^{\prime}\left(a^{\prime}, u^{\prime}\right)=\left(\operatorname{det} u^{\prime}\right)^{-\frac{1}{2}} \exp \left(-a^{\prime}+\gamma \operatorname{tr} u^{\prime}\right) \operatorname{det} \Gamma\left(1+u^{\prime}\right)
$$

Note that we have also used $\operatorname{det} M=\exp (\operatorname{tr} \ln M)$ to get (2.8).

To complete the story, let us look more closely at what happens within other regularizations. The regularization that appeared in [2] looks a little bit cumbersome for the problem of interest. Moreover, it assumes that the boundary propagator (1.6) is modified to

$$
\left\langle X^{\mathrm{T}}(\theta) X\left(\theta^{\prime}\right)\right\rangle=\frac{2}{t}+2 \sum_{k=1}^{\infty} \frac{\mathrm{e}^{i k\left(\theta-\theta^{\prime}\right)}}{k+(t+k f) \mathrm{e}^{-\epsilon k}}+\frac{\mathrm{e}^{-i k\left(\theta-\theta^{\prime}\right)}}{k+(t-k f) \mathrm{e}^{-\epsilon k}},
$$

where $\epsilon$ is the short distance cut-off. It is clear that at vanishing $t$ and $f$ the propagator (2.9) becomes divergent. It poses a natural question whether such a divergence is relevant or not $\$$. Instead of answering this question, let us propose another regularization which avoids the latter problem. Moreover, it seems a little bit simpler for explicit calculations. In this regularization scheme ? the propagator is given by

$$
\left\langle X^{\mathrm{T}}(\theta) X\left(\theta^{\prime}\right)\right\rangle=\frac{2}{t}+2 \sum_{k=1}^{\infty} \mathrm{e}^{-\epsilon k}\left(\frac{\mathrm{e}^{i k\left(\theta-\theta^{\prime}\right)}}{k+\mathbf{t}+k \mathbf{f}}+\frac{\mathrm{e}^{-i k\left(\theta-\theta^{\prime}\right)}}{k+\mathbf{t}-k \mathbf{f}}\right),
$$

Now the equations (1.5) can be solved by

$$
Z(\mathbf{a}, \mathbf{t}, \mathbf{f}, \epsilon)=(\operatorname{det} \mathbf{t})^{-\frac{1}{2}} \mathrm{e}^{-\mathbf{a}} \exp \left(-\sum_{k=1}^{\infty} \mathrm{e}^{-\epsilon k} \operatorname{tr} \ln \left(1+k^{-1} \mathbf{t}+\mathbf{f}\right)\right) .
$$

The preceding calculations can be generalized without difficulty to this case $\square$. The partition function takes the form

$$
Z(\mathbf{a}, \mathbf{t}, \mathbf{f}, \epsilon)=(\operatorname{det} \mathbf{u})^{-\frac{1}{2}} \exp \left(-\mathbf{a}+\ln \epsilon \operatorname{tr} \mathbf{u}-\frac{1}{\epsilon} \operatorname{tr} \ln (1+\mathbf{f})+\sum_{n=2}^{\infty} \frac{(-)^{n}}{n} \zeta(n) \operatorname{tr} \mathbf{u}^{n}+O(\epsilon)\right)
$$

\footnotetext{
${ }^{8}$ In fact, the regularization prescription of Li-Witten is more involved. It includes nonlocal boundary interactions that do not lead to short distance divergencies, so one does not need the regularized propagator for vanishing $t$ and $f$. However at some point it assumes that the interactions become local that can run into difficulty.

${ }^{9}$ In recognition of this, we henceforce denote the parameters as $(\mathbf{a}, \mathbf{t}, \mathbf{f})$. For zero value of the tachyon field, this regularization was proposed in 17, 13.

${ }^{10}$ Some useful formulae can be found in appendix of 13 .
} 
in which $\mathbf{u}=\frac{1}{1+\mathbf{f}} \mathbf{t}$. Before we start a comparison of the above result and the result we obtained in the framework of the $\zeta$-function regularization, let us make a simple remark. In leading order, $\zeta(1)$ may be identified with $-\ln \epsilon$ according to $\sum_{n=1}^{\infty} \frac{1}{n} \mathrm{e}^{-\epsilon n}=-\ln \epsilon+O(\epsilon)$. Thus the main novelty which is now visible in Eq.(2.12) is the appearance of the power divergence. We again use the minimal subtraction to renormalize the partition function 17

$$
\mathbf{a}=\mathbf{a}^{\prime}+\ln \epsilon \operatorname{tr} \mathbf{u}^{\prime}-\frac{1}{\epsilon} \operatorname{tr} \ln \left(1+\mathbf{f}^{\prime}\right) \quad, \quad \mathbf{u}=\mathbf{u}^{\prime} \quad, \quad \text { with } \quad \mathbf{t}=\mathbf{t}^{\prime} \quad, \quad \mathbf{f}=\mathbf{f}^{\prime} .
$$

As a result, we have

$$
Z^{\prime}\left(\mathbf{a}^{\prime}, \mathbf{u}^{\prime}\right)=\left(\operatorname{det} \mathbf{u}^{\prime}\right)^{-\frac{1}{2}} \exp \left(-\mathbf{a}^{\prime}+\gamma \operatorname{tr} \mathbf{u}^{\prime}\right) \operatorname{det} \Gamma\left(1+\mathbf{u}^{\prime}\right)
$$

In fact, the renormalization as it is done in above is incomplete. The missing point is the renormalization conditions which fix the finite part of $Z^{\prime}$ removing ambiguities due to a particular renormalization scheme. Unfortunately, it is not known what these conditions are. Unfortunately, we don't have something new to say here. Nevertheless, a natural question which comes to mind to address is whether something universal can be found within at least two regularization schemes we use. It turns out that this question has a natural answer. First, let us note that under a scale transformation $\epsilon \rightarrow \lambda \epsilon$, the expressions (2.3) and (2.12) transform as 12

$$
Z(\mathrm{a}, \mathrm{t}, \mathrm{f}) \rightarrow \mathrm{e}^{\ln \lambda \operatorname{tru}} Z(\mathrm{a}, \mathrm{t}, \mathrm{f})
$$

where $\mathrm{a}=(a, \mathbf{a}), \mathrm{t}=(t, \mathbf{t}), \mathrm{f}=(f, \mathbf{f})$, here and below. By using the minimal subtraction, the renormalized partition function takes the form

$$
Z^{\prime}\left(\mathrm{a}^{\prime}, \mathrm{t}^{\prime}, \mathrm{f}^{\prime}\right)=\left(\operatorname{det} \mathrm{u}^{\prime}\right)^{-\frac{1}{2}} \exp \left(-\mathrm{a}^{\prime}+c \operatorname{tr} \mathrm{u}^{\prime}\right) \operatorname{det} \Gamma\left(1+\mathrm{u}^{\prime}\right)
$$

with an arbitrary constant $c$. This implies that the partition function is a scheme-dependent object. Note that it makes no sense to set $c$ equal to a certain number as far as the normalization conditions are missing.

Before continuing our discussion, we will make a detour and rederive some basic results on the $\mathrm{RG} \beta$-functions in the problem of interest. To do so, let us introduce an arbitrary quantity $\mu$ with the dimension of a mass with respect to the world-sheet theory and some ultraviolet cut-off $\Lambda$. Then, Eq.(2.13) can be rewritten as

$$
\frac{\mathbf{a}}{\mu}=\mathbf{a}^{\prime}+\ln \frac{\mu}{\Lambda} \operatorname{tr} \mathbf{u}^{\prime}-\frac{\Lambda}{\mu} \operatorname{tr} \ln \left(1+\mathbf{f}^{\prime}\right) \quad, \quad \frac{\mathbf{t}}{\mu}=\mathbf{t}^{\prime} \quad, \quad \mathbf{f}=\mathbf{f}^{\prime} \quad,
$$

in which $\epsilon=\frac{\mu}{\Lambda}$ and all renormalized couplings are explicitly dimensionless with respect to the worldsheet theory.

It is well-known that the scale transformations $\mu \rightarrow \mu(1+\sigma)$ with an infinitesimal parameter $\sigma$ result in the corresponding shifts of the renormalized couplings i.e., $\mathrm{x}^{i} \rightarrow \mathrm{x}^{i}+\sigma \beta^{i}$. The function $\beta^{i}$ is called the RG $\beta$-function. A simple algebra shows that in the problem at hand

$$
\beta_{\mathbf{a}}=-\operatorname{tr} \mathbf{u}^{\prime}-\mathbf{a}^{\prime} \quad, \quad \beta_{\mathbf{t}}=-\mathbf{t}^{\prime} \quad, \quad \beta_{\mathbf{f}}=0 .
$$

It is a straightforward matter of working out the calculations within the $\zeta$-function regularization. The result is the same as in above.

\footnotetext{
${ }^{11}$ See also $[17]$.

${ }^{12}$ Here we assume that $\zeta(1)=-\ln \epsilon+O(\epsilon)$.
} 
Now consider RG fixed points which are defined by $\beta_{\mathrm{x}}=0$ [5]. It is easy to see at least one family of the solutions

$$
\mathrm{a}^{\prime}=0 \quad, \quad \mathrm{t}^{\prime}=0 \quad, \quad \mathrm{f}^{\prime}=\text { fixed }
$$

that is parameterized by the antisymmetric tensor $\mathrm{f}^{\prime}$.

It is also easy to see that at the vicinity of these solutions the partition function shows the critical behavior. Indeed, for example setting t' to be diagonal $\mathrm{t}^{\prime}=\operatorname{diag}\left(t_{1}, \ldots, t_{n}\right)$, one finds

$$
Z^{\prime}\left(\mathrm{a}^{\prime}, \mathrm{t}^{\prime}, \mathrm{f}^{\prime}\right) \sim \prod_{i=1}^{n} t_{i}^{-\frac{1}{2}} \quad, \quad \text { as } \quad t_{i} \rightarrow 0
$$

Since it is usual to say that the leading asymptotic of the partition function at the vicinity of the critical points should be universal we have at least one explicit asymptotics in the problem of interest

$$
Z^{\prime}\left(\mathrm{a}^{\prime}, \mathrm{t}^{\prime}, \mathrm{f}^{\prime}\right)=\left(\operatorname{det} \mathrm{u}^{\prime}\right)^{-\frac{1}{2}} \mathrm{e}^{-\mathrm{a}^{\prime}} \text { for } \mathrm{t}^{\prime} \sim 0
$$

At this point we should stress that we relax the requirement $\mathrm{a}^{\prime} \sim 0$ and include the factor $\mathrm{e}^{-\mathrm{a}^{\prime}}$ in $Z^{\prime}$. Motivations for doing so come from our main suggestion which is to replace unknown normalization conditions by universality. Indeed, Eq.(2.16) shows that such a factor is universal while the ambiguity due to $c$ drops from the above asymptotics that is in agreement with our expectations.

Let us conclude this subsection by making some remarks.

(i) Our result (2.16) passes through the requirement of Ref. [2] which in the case of interest is

$$
\left.Z^{\prime}\left(\mathrm{a}^{\prime}, \mathrm{t}^{\prime}, \mathrm{f}^{\prime}\right)\right|_{\mathrm{f}^{\prime}=0}=Z^{\prime}\left(\mathrm{a}^{\prime}, \mathrm{t}^{\prime}\right) \text {. }
$$

This fact is evident since the renormalized couplings for the tachyon and gauge field enter only through a special combination $\frac{1}{1+\mathrm{f}^{\prime}} \mathrm{t}^{\prime}$. So, Eq. 2.22) can not be considered as complete renormalization conditions.

(ii) A simple algebra show that the result (2.16) is in a complete agreement with earlier computations [15, 18]. Indeed, setting $\mathrm{t}^{\prime}=0$ one finds that the only $\mathrm{f}^{\prime}$-dependent contribution comes from the first factor and it is $\sqrt{\operatorname{det}\left(1+f^{\prime}\right)}$. The divergence due to det $t^{\prime}$ is related to the fact that in this case the fields $X^{\mu}$ have zero modes and must be interpreted as the space-time volume (see [2] for details). As a result, $Z^{\prime}$ provides the Born-Infeld action.

(iii) There exists another interesting possibility to fulfill the requirement of universality. To see what happens, let us introduce a general constant space-time metric $g_{i j}$ for the bulk action (1.2). This results in a minor modification of all story as $\mathrm{u}$ now becomes $\mathrm{u}=\frac{1}{g+\mathrm{f}} \mathrm{t}$. For $g=0$, we have $\operatorname{tr} \mathrm{u} \equiv 0$ that allows us to conclude that $c$ in (2.16) drops out. So, it makes sense to keep all terms in the expression (2.16). Using

$$
\ln \Gamma(1+M)=\frac{1}{2} \ln \left(\frac{\pi M}{\sin \pi M}\right)-\sum_{n=1}^{\infty} \frac{1}{2 n+1} \zeta(2 n+1) M^{2 n+1}-\gamma M \quad,
$$

that is a matrix generalization of the identity for the logarithm of the $\gamma$-function [16], we end up with the following expression for the partition function 14

$$
Z^{\prime}\left(\mathrm{a}^{\prime}, \mathrm{t}^{\prime}, \mathrm{f}^{\prime}\right)=\left(\operatorname{det} \sin \left(\pi \frac{1}{\mathrm{f}^{\prime}} \mathrm{t}^{\prime}\right)\right)^{-\frac{1}{2}} \exp \left(-\mathrm{a}^{\prime}\right) \quad .
$$

At this point, it is not difficult to see some analogy with the Seiberg-Witten description of open string theory in the presence of a constant $B$-field 19. Indeed, we have for the open string metric and parameter of noncommutativity $G^{-1}=0$ and $\theta_{n}=\frac{1}{B}=\frac{1}{\mathrm{f}^{\prime}}$, respectively. However we do not take the $\alpha^{\prime} \rightarrow 0$ as we wish to hold $\alpha^{\prime}$-dependence.

\footnotetext{
${ }^{13}$ We do not consider $\beta_{\mathrm{x}}=\infty$.

${ }^{14}$ We omit overall numerical factors in expressions for the partition functions as they have no meaning.
} 


\subsection{The fermionic string}

We will now extend our analysis to the problem of the evaluation of the partition function, including the integration over fermions. As we've already emphasized the expression (1.19) is purely formal since it may be divergent. To make sense of the partition function, one has to introduce a regularization procedure. Following subsection 2.1, we begin with the $\zeta$-function regularization scheme.

First, we rewrite (1.19) as

$$
\begin{aligned}
\hat{Z}(a, t, f)= & (\operatorname{det} t)^{-\frac{1}{2}} \exp \left(-a+\left[\zeta\left(0, \frac{1}{2}\right)-\zeta(0)\right] \operatorname{tr} \ln (1+f)\right) \\
& \times \exp \left(-\sum_{k=1}^{\infty} \operatorname{tr} \ln \left(1+k^{-1} \frac{1}{1+f} t\right)+\sum_{r=\frac{1}{2}}^{\infty} \operatorname{tr} \ln \left(1+r^{-1} \frac{1}{1+f} t\right)\right) .
\end{aligned}
$$

Expanding the logarithms in power series and collecting terms, one finds

$$
\begin{aligned}
\hat{Z}(a, t, f)= & (\operatorname{det} t)^{-\frac{1}{2}} \exp \left(-a+\left[\zeta\left(0, \frac{1}{2}\right)-\zeta(0)\right] \operatorname{tr} \ln (1+f)+\left[\zeta\left(1, \frac{1}{2}\right)-\zeta(1)\right] \operatorname{tr} \frac{1}{1+f} t\right) \\
& \times \exp \left(\sum_{n=2}^{\infty} \frac{(-)^{n}}{n}\left[\zeta(n)-\zeta\left(n, \frac{1}{2}\right)\right] \operatorname{tr}\left(\frac{1}{1+f} t\right)^{n}\right) .
\end{aligned}
$$

Finally, using $\zeta\left(n, \frac{1}{2}\right)=\left(2^{n}-1\right) \zeta(n)$ and introducing $u$, we get

$$
\hat{Z}(a, u)=(\operatorname{det} u)^{-\frac{1}{2}} \exp \left(-a+\sum_{n=2}^{\infty} \frac{(-)^{n}}{n}\left(2-2^{n}\right) \zeta(n) \operatorname{tr} u^{n}\right) .
$$

The main novelty now is that the partition function became finite. So, we do not need to subtract the logarithmic divergence anymore. We postpone the introduction of the renormalized couplings for a moment. At this point a comment is in order.

Using (2.6), a small computation shows that the fermionic and bosonic partition functions obey

$$
\hat{Z}(a, u)=\frac{\left(Z^{\prime}(a, u)\right)^{2}}{Z^{\prime}(a, 2 u)} .
$$

This is different from what is recently found in [7]. The difference is due to a factor $4^{\operatorname{tru}}$. Due to the appearance of $\operatorname{tr} u$ it immediately comes to mind to make a double check by using another regularization scheme as the term with tru was the origin of all our troubles in subsection 2.1.

Let us compute the partition function within a regularization scheme which contains an explicit short distance cut-off $\epsilon$. For our purposes it turns out enough to define the regularized propagator for the $X$ 's as (2.10) while for the fermions as

$$
\left\langle\psi^{\mathrm{T}}(\theta) \psi\left(\theta^{\prime}\right)\right\rangle=2 i \sum_{r=\frac{1}{2}}^{\infty} \mathrm{e}^{-\epsilon r}\left(\frac{r \mathrm{e}^{i r\left(\theta-\theta^{\prime}\right)}}{r+\mathbf{t}+r \mathbf{f}}-\frac{r \mathrm{e}^{-i r\left(\theta-\theta^{\prime}\right)}}{r+\mathbf{t}-r \mathbf{f}}\right) .
$$

Now the equations (1.16)-(1.17) can be solved by

$$
\hat{Z}(\mathbf{a}, \mathbf{t}, \mathbf{f}, \epsilon)=(\operatorname{det} \mathbf{t})^{-\frac{1}{2}} \mathrm{e}^{-\mathbf{a}} \exp \left(-\sum_{k=1}^{\infty} \mathrm{e}^{-\epsilon k} \operatorname{tr} \ln \left(1+k^{-1} \mathbf{t}+\mathbf{f}\right)+\sum_{r=\frac{1}{2}}^{\infty} \mathrm{e}^{-\epsilon r} \operatorname{tr} \ln \left(1+r^{-1} \mathbf{t}+\mathbf{f}\right)\right) .
$$


The preceding calculations can be generalized without difficulty to this regularization scheme. So we have

$$
\hat{Z}(\mathbf{a}, \mathbf{u}, \epsilon)=(\operatorname{det} \mathbf{u})^{-\frac{1}{2}} \exp \left(-\mathbf{a}+\ln 4 \operatorname{tr} \mathbf{u}+\sum_{n=2}^{\infty} \frac{(-)^{n}}{n}\left(2-2^{n}\right) \zeta(n) \operatorname{tr} \mathbf{u}^{n}\right)
$$

Thus the partition function is again finite but it differs from the former one by a factor $4^{\operatorname{tr}} \mathbf{u}$. On the one hand, it sounds good as it is in harmony with the result of [7]. On the other hand, it poses a natural question about universality we have already discussed in subsection 2.1 as a general form of $\hat{Z}$ turns out to be

$$
\hat{Z}(\mathrm{a}, \mathrm{u})=(\operatorname{det} \mathrm{u})^{-\frac{1}{2}} \exp \left(-\mathrm{a}+\hat{c} \operatorname{tru}+\sum_{n=2}^{\infty} \frac{(-)^{n}}{n}\left(2-2^{n}\right) \zeta(n) \operatorname{tr} \mathrm{u}^{n}\right),
$$

where $\hat{c}$ is an arbitrary constant.

It seems reasonable to set $\mathrm{a}=0$ as this coupling has no meaning in the fermionic case. Now, Eq.(2.17) is modified to

$$
\frac{\mathrm{t}}{\mu}=\mathrm{t}^{\prime} \quad, \quad \mathrm{f}=\mathrm{f}^{\prime}
$$

that still allows non-trivial $\beta$-function

$$
\beta_{\mathrm{t}}=-\mathrm{t}^{\prime} \quad, \quad \beta_{\mathrm{f}}=0
$$

The RG fixed points are again a family parameterized by antisymmetric tensor (see (2.19)).

As the asymptotics at the vicinity of the critical points is believed to be universal we get

$$
\hat{Z}\left(\mathrm{u}^{\prime}\right) \sim\left(\operatorname{det} \mathrm{u}^{\prime}\right)^{-\frac{1}{2}} \quad, \quad \text { for } \quad \mathrm{t}^{\prime} \sim 0,
$$

that is in harmony with our expectations as $\hat{c}$ drops out.

At this point, let us makes some remarks.

(i) As in the bosonic case, our result for the partition function 2.32 simply passes through the requirement like (2.22). Thus, the use of that as the renormalization conditions fails.

(ii) As we have discussed at the end of the previous subsection, the asymptotics like (2.35) or expressions like (2.32) at $\mathrm{t}=0$ recover the Born-Infeld action. So, there is a complete agreement with earlier computations [20, 21].

(iii) In the case of a general space-time metric $g$, we can take $g=0$. If we do so, then the origin of our troubles, tr $\mathrm{u}$, identically equals zero. With such a good novelty we can hold all terms in (2.32). Some evidence that it is a right way comes from our results and from a general consideration within the perturbation theory which shows that these terms appear by finite integrals. Thus the partition function becomes

$$
\hat{Z}\left(\mathrm{a}^{\prime}, \mathrm{t}^{\prime}, \mathrm{f}^{\prime}\right)=\left(\operatorname{det} \tan \left(\pi \frac{1}{\mathrm{f}^{\prime}} \mathrm{t}^{\prime}\right)\right)^{-\frac{1}{2}}
$$

To get this form we used the matrix relations: $\sin 2 M=2 \sin M \cos M$ and $\tan M=\sin M / \cos M$. 


\section{$3 \quad$ String Field Actions}

Now we come to a key point. What is the background independent string field theory action in which we can belive? If the normalization conditions are missing, it makes no sense to rigorously speak about $S$. Nevertheless, we will try to do so. Our main requirement which replaces the normalization conditions will be universality. In other words, we believe in structures which are independent of renormalization schemes for the world-sheet theories.

In studying the BIOST actions, we begin with the bosonic string. The basic fact we've learned in section 2 is that it is a pointless deal to use the expression of $Z^{\prime}$ computed in a special regularization scheme for lack of universality. Instead of this, we can believe at least in the asymptotics (2.21). As a result, all linear terms in $\mathrm{t}^{\prime}$ can be dropped in deriving the asymptotics of the background independent string field theory action for $\mathrm{t}^{\prime} \sim 0$. This leads to a significant simplification in the integration of the partial differential equations (1.8). Moreover, as we will see in a moment it avoids the problem we mentioned in introduction namely, why $S_{w}$ is better than $S_{s}$ and vice versa.

We are now ready to go. The simplest way to get what we need is to consider the $\zeta$-function regularization scheme. So, taking

$$
d O(\theta)=c\left(\frac{1}{2 \pi} d \mathrm{a}+\ldots\right)(\theta)
$$

together with

$$
\{Q, O\}\left(\theta^{\prime}\right)=c \dot{c}\left(\frac{1}{2 \pi} a+\frac{1}{8 \pi} X^{\mathrm{T}} t X \ldots\right)\left(\theta^{\prime}\right)
$$

where dots mean terms that are irrelevant, we immediately get the equation

$$
\frac{\partial S_{w}}{\partial a}=\left(-a+t \frac{\partial}{\partial t}\right) Z
$$

In our approximation, it can be solved by

$$
S_{w}=\left(1+a-t \frac{\partial}{\partial t}\right) Z
$$

Using the expression (2.3), we see that $a$ can be renormalized as (2.5). Thus the background independent string field theory action is a function of the renormalized couplings. At this point a couple of remarks is in order.

(i) Note that the right hand side of Eq.(3.2) is due to the ghosts contractions, so it does not depends on the details of the matter sector, namely whether the BRST charge depends on the couplings or not. From this point of view it is universal.

(ii) In general, it is a non-trivial consistency check to see that the BIOST action is a function on the renormalized couplings. In the framework of another regularization scheme it was shown in [2]. Thus, it appears that this is the case.

Finally, we have the following asymptotics for the BIOST action

$$
S_{w}=\left(\operatorname{det} \mathrm{u}^{\prime}\right)^{-\frac{1}{2}}\left(1+\mathrm{a}^{\prime}+\frac{D}{2}\right) \mathrm{e}^{-\mathrm{a}^{\prime}} \text { for } \quad \mathrm{t}^{\prime} \sim 0 .
$$

Now let us try to see what happens with the action $S_{s}$ defined in (1.10). Borrowing our result for the RG $\beta$-functions (2.18) from section 2, we immediately get the same expression as above. Thus 
we conclude that the universal structure of $S_{w}$ and $S_{s}$ is the same i.e., from our point of view there is no difference between these actions. At this point, a natural question to ask is what to do with non-universal structures that are responsible for difference. Unfortunately, we do not know what the exact answer is. We can only suggest that these structures may be transformed each into another under a set of coupling's redefinitions.

To compare our result with "experiment", we follow the lines of [6]. So, we take the effective action for the tachyon and gauge field

$$
S_{e f f}=\int d^{D} x \sqrt{\operatorname{det}(1+F)} \mathrm{e}^{-T}(1+T+O(\partial T, \partial F)),
$$

where $O(\partial T, \partial F)$ means derivative terms. Next we evaluated it for the profiles whose forms are motivated by Eq.(1.3)

$$
T(X)=\mathrm{a}^{\prime}+X^{\mathrm{T}} \mathrm{t}^{\prime} X \quad, \quad F(X)=\mathrm{f}^{\prime} .
$$

A simple algebra shows that the result is given by (3.5) that is exactly what we need. Note that for $F=0$ the same potential was obtained in [5, 6. Moreover, it has recently appeared in [14].

Note that we have not included the derivative terms in (3.6). The latter is meaningless as this would lead to $t^{\prime}$ corrections which are over control in the framework of the background independent string field theory we consider. Thus it makes no sense to speculate about the tachyon mass. We do not control it. What we certainly control is the only potential that is related with the universal structure.

To conclude our discussion of bosonic string, let us remark that the expression (2.24) in fact leads to the tachyon potential within the noncommutative scalar field theory whose parameter of noncommutativity $\theta_{n}=\frac{1}{f^{\prime}}$. A formal proof is straightforward: first we use it to compute $S$; next we go along the lines of 19] 四. The only difference is that in our case $G^{-1}=0$ while $\theta_{n}$ is arbitrary. The first allows us to suppress all derivative terms. As to the second, it allows us to avoid taking a large noncommutativity limit that shows that the form of potential is universal i.e., it is the same for all $\theta_{n}\left(\mathrm{f}^{\prime}\right)$.

We now turn to the problem of shedding some light on the boundary string field theory action in the fermionic case. As we've already mentioned the fermionic counterpart of the Witten's action (1.8) is still missing, so we suggested to define the action as (1.20). Our motivation to do so is the following: as we've seen in section 2 , there is no difference between two actions $S_{w}$ and $S_{s}$ from our point of view i.e., universality. So, if the definition of $\hat{S}_{w}$ in the framework of the Batalin-Vilkovisky formalism is absent, then we can use our definition of $\hat{S}_{s}$. The latter is based on a natural supersymmetrization of $S_{s}$.

From (2.34) and (2.35), we get

$$
\hat{S}_{s} \sim\left(\operatorname{det} \mathrm{u}^{\prime}\right)^{-\frac{1}{2}}\left(1+\frac{D}{2}\right) \quad, \quad \text { for } \quad \mathrm{t}^{\prime} \sim 0,
$$

that formally coincides with the bosonic action $(3.5)$ evaluated at $\mathrm{a}^{\prime}=0$.

The comparison of the above result with "experiment" is straightforward. Taking the effective action

$$
\hat{S}_{e f f}=\int d^{D} x \sqrt{\operatorname{det}(1+F)} \mathrm{e}^{-T^{2}}\left(1+T^{2}+O(\partial T, \partial F)\right),
$$

and evaluating it for the linear profiles ${ }^{16}$

$$
T(X)=u X \quad \text { with } \quad \mathrm{t}^{\prime}=u^{2} \quad ; \quad F(X)=\mathrm{f}^{\prime} \quad,
$$

\footnotetext{
${ }^{15}$ See also [22, 14 for more details.

${ }^{16} \mathrm{~A}$ constant a has been omitted, because it can be absorbed in a shift in the $X$ 's (as long as $u \neq 0$ ). This is in a complete agreement with our conclusions of section 2 where we dropped a' based on the RG analysis.
} 
we immediately get a desired result (3.8).

On the other hand, the definition (1.21) gives simply

$$
\hat{S} \sim\left(\operatorname{det} \mathrm{u}^{\prime}\right)^{-\frac{1}{2}} \quad, \quad \text { for } \quad \mathrm{t}^{\prime} \sim 0
$$

So the effective action is now $\square$

$$
\hat{S}_{e f f}=\int d^{D} x \sqrt{\operatorname{det}(1+F)} \mathrm{e}^{-T^{2}}(1+O(\partial T, \partial F)) .
$$

We should noted that for $F=0$ the same result has been recently found in [7].

At first glance, the expressions (3.9) and (3.12) are not equivalent. However, because we omit the derivative terms we can transform the actions each into another by a field redefinition. Moreover, the potentials have a similar profile with the same extrema at $T_{\text {ext }}=\{0, \infty\}$. So, from this point of view we can not say that the expressions are different. Our conclusion is that the factor $\sqrt{\operatorname{det}(1+F)} \mathrm{e}^{-T^{2}}$ in the effective actions is universal. Unfortunately, we have no arguments in our disposal to say why $\hat{S}_{s}$ is better than $\hat{S}$ or vice versa.

As in the bosonic case, we do not include the derivative terms in $(3.9),(3.12)$ because they lead to $\mathrm{t}^{\prime}$ corrections which are over control. Thus it makes no sense to speculate about the tachyon mass. We only control the potential that is related with the universal structure.

To conclude our discussion of fermionic string, let us discuss the expression (2.36) more carefully than we have done that for its counterpart in the case of bosonic string where the expression (2.24) leaded us to the potential of the noncommutative scalar field theory. Indeed, our discussion was a little bit cavalry. The point is that noncommutative field theories appear from open string in the presence of a constant $B$-field only within a very special regularization scheme, the so-called point splitting regularization [19] while we got the noncommutative scalar field theory for all our schemes. Moreover, this was also the case in [22, 14. Examples with the tachyon vertex operator are in some sense confusing as any regularization scheme gives the $*$-product structure ${ }^{10}$. To be more precise, let us recall two famous facts: first, the bosonic tachyon vertex operator is simply $\mathrm{e}^{i k X}$; second, the propagator of $X$ 's evaluated at the boundary of the upper half plane is given by

$$
\left\langle X^{\mathrm{T}}(\tau) X\left(\tau^{\prime}\right)\right\rangle=-2 G^{-1} \ln \left(\tau-\tau^{\prime}\right)^{2}+2 \pi i \theta_{n} \varepsilon\left(\tau-\tau^{\prime}\right) .
$$

In our notations, $G=1-f^{2}, \theta_{n}=f / 1-f^{2}$ (see (1.6)). Thus, every time $\theta_{n}$ appears in a such way that the $*$-product structure is correct. However, we run into difficulty as soon as we would like to consider other vertex operators, for example, the gauge field vertex operator. The known remedy is the use of the point splitting regularization. Now let us see what happens in the fermionic case. This time the tachyon vertex operator ${ }^{19}$ gets a contribution like $k \psi \partial_{\tau}^{-1} k \psi \mathrm{e}^{i k X}$ by the fermions $\psi$ 's whose propagator evaluated at the boundary of the upper half plane is

$$
\left\langle\psi^{\mathrm{T}}(\tau) \psi\left(\tau^{\prime}\right)\right\rangle=4 G^{-1} \frac{1}{\tau-\tau^{\prime}}-4 \pi i \theta_{n} \delta\left(\tau-\tau^{\prime}\right)
$$

It is clear that the last term in (3.14) clashes with noncommutativity since $\theta_{n}$ can appear not only due to the $*$-product. The remedy is again the use of the point splitting regularization that automatically fixes the problem. After this is understood, it becomes clear that it makes no sense to look for noncommutative field theory to compare its action with the action coming from the use of the expression

\footnotetext{
${ }^{17}$ For $F=0$, this action has been proposed based on the exactly solvable Schroedinger problem [9].

${ }^{18}$ We refer to [19] for a review of noncommutative geometry within string theory.

${ }^{19}$ We mean the operator that follows from the boundary action $(1.13)$.
} 
(2.36). First, we have to get rid of the fermionic contribution. It is clear that this brings the result to the bosonic form (2.24). So, for example, using the definition (1.21), we get

$$
\hat{S}_{e f f}=\int d^{D} x * \mathrm{e}^{-T^{2}}
$$

We do not include derivative terms as $G^{-1}=0$.

\section{Conclusions and Remarks}

First let us say a few words about the results.

In this work we have discussed what information can be safety extracted from background independent off-shell string theory in the case of missing renormalization conditions for the underlying world-sheet theories. We propose a principle of universality to somehow compensate this drawback. It states that one believes in structures in BIOST actions which are independent of a renormalization scheme used for the world-sheet theories. To do so, we considered the tachyon and gauge fields backgrounds and carried out computations in the different renormalization schemes for both, bosonic and fermionic strings. In the latter case, we also proposed the definition of the BIOST action since the rigorous definition based on the Batalin-Vilkovisky formalism is still missing in this case. Our main conclusion is that some asymptotics which are responsible for the potentials only obey the principle of universality.

Let us conclude by making a couple of remarks.

(i) All the results for the potentials can be easily obtained in the framework of the so-called sigma model approach to string theory 20 equipped with the definitions of the actions. Let us briefly illustrate how this goes in the case of superstring. Splitting the integration variable $X$ in the constant and the non-constant part as $X(\theta)=x+\xi(\theta)$ and dropping all derivative terms like $\partial T, \partial F$ from the boundary interactions

$$
\hat{S}_{b}=\oint_{\partial D} d \theta\left(T^{2}(X)+\psi \partial T \partial_{\theta}^{-1} \psi \partial T(X)+i \dot{X} A(X)+\frac{i}{2} \psi^{\mathrm{T}} F(X) \psi\right),
$$

we immediately get for the partition function

$$
\hat{Z} \sim \int d^{D} x \sqrt{\operatorname{det}(1+F)} \mathrm{e}^{-T^{2}}
$$

where the Born-Infeld factor is due to the integration over $\xi$ [21] and $T$ is real. Thus, in the case $\hat{S}=\hat{Z}$ we recover the result (3.12). On the other hand,

$$
\beta_{T}=-\frac{1}{2} T+O(\partial T, \partial F) \quad, \quad \beta_{A}=0+O(\partial T, \partial F)
$$

together with the definition $(1.20)$ exactly result in Eq.(3.9) i.e., what we got in section 3. The expressions for the $\beta$-functions follow from our result (2.34). For example, substituting $\mathrm{t}^{\prime}=u^{2}$ into the first equation of (2.34), we get $\beta_{u}=-\frac{1}{2} u$ which for the linear tachyon profile (3.10) provides a desired result.

On the one hand, an apparent advantage of this approach with respect to BIOST is that it works for any profile of the tachyon field 27. On the other hand, BIOST turns out to be much simpler to analyze ambiguity related with derivative terms.

\footnotetext{
${ }^{20}$ See the last reference in $\sqrt{10}$ for a review.

${ }^{21}$ In fact, it was used in 22 to derive the potential in the case of bosonic string with a large $B$-field.
} 
(ii) The main motivation in studying the tachyon potentials is the phenomenon of tachyon condensation [23]. It has been recently realized that BIOST turns out to be very useful to get a correct energetics of this phenomenon [6, 11]. We are not going to discuss all related issues here as it is out of scope of the present paper but there is one which we would like to mention. It is clear from our analysis that the actions as well as partition functions depend on a universal variable $u=\frac{1}{1+\mathrm{f}} \mathrm{t}$ rather than $\mathrm{t}$ and $\mathrm{f}$. If one calls $\mathrm{a}=\infty, \mathrm{t}=0$ as the vacuum configuration [11], then $\mathrm{f}=$ fixed is irrelevant for this configuration. This is in harmony with the Sen conjecture that at the minimum of the tachyon potential all different values of the gauge field strength $F$ describe the same physics 24 .

\section{Acknowledgments}

We would like to thank A.A. Tseytlin for a collaboration at an initial stage, and also for useful comments. We are also grateful to B. Zwiebach for helpful discussions and H. Dorn for reading the manuscript. The work is supported in part by DFG under Grant No. DO 447/3-1 and the European Community grant INTAS-OPEN-97-1312.

\section{References}

[1] E. Witten, Phys.Rev. D46 (1992) 5467; Phys.Rev. D47 (1993) 3405.

[2] K. Li and E. Witten, Phys.Rev. D48 (1993) 853.

[3] S. L. Shatashvili, Phys.Lett. B311 (1993) 83.

[4] S. L. Shatashvili, "On the Problems with Background Independence in String Theory", Report No. IASSNSHEP-93/66, hep-th/9311177.

[5] A. A. Gerasimov and S. Shatashvili, "On exact tachyon potential in open string theory", Report No. YCTPP9-00, hep-th/0009103.

[6] D. Kutasov, M. Marino, and G. Moore, "Some exact results on tachyon condensation in string field theory", Report No. EFI-2000-32, hep-th/0009148.

[7] D. Kutasov, M. Marino, and G. Moore, "Remarks on Tachyon Condensation in Superstring Theory", Report No. EFI-2000-40, hep-th/0010108.

[8] J. A. Minahan and B. Zwiebach, "Field theory models for tachyon and gauge field string dynamics", Report No. CTP-MIT-3019, hep-th/0008231.

[9] J. A. Minahan and B. Zwiebach, "Effective Tachyon Dynamics in Superstring Theory", Report No. CTPMIT-3027, hep-th/0009246.

[10] C. Lovelace, Nucl.Phys. B273 (1986) 413;

T. Banks and E. Martinec, Nucl.Phys. B294 (1987) 733;

I. Klebanov and L. Susskind, Phys.Lett. B200 (1988) 446;

A. A. Tseytlin, Int.J.Mod.Phys. A4 (1989) 1257.

[11] D. Ghoshal and A. Sen, "Normalization of the Background Independent Open String Field Theory Action", Report No. MRI-P-000905, hep-th/0009191.

[12] E. Witten, JHEP 9812 (1998) 019.

[13] O. D. Andreev and A. A. Tseytlin, Nucl.Phys. B311 (1988) 205.

[14] K. Okuyama," Noncommutative Tachyon from Background Independent Open String Field Theory", Report No. KEK-TH-719, hep-th/0010028.

[15] E. S. Fradkin and A. A. Tseytlin, Phys.Lett. B163 (1985) 123.

[16] I. S. Gradshteyn and I. M. Ryzhik, Table of Integrals, Series, and Products (Academic Press, 1994). 
[17] A. A. Tseytlin, Phys.Lett. B202 (1988) 81.

[18] A. Abouelsaood, C. G. Callan, C. N. Nappi, and S. A. Yost, Nucl.Phys. B280 (1987) 599.

[19] N. Seiberg and E. Witten, JHEP 9909 (1999) 032.

[20] E. Bergshoeff, E. Sezgin, C. N. Pope, and P. K. Townsend, Phys.Lett. B188 (1987) 70.

[21] R. R. Metsaev, M. A. Rakhmanov, and A. A. Tseytlin, Phys.Lett. B193 (1987) 207.

[22] L. Cornalba, "Tachyon Condensation in Large Magnetic Fields with Background Independent String Field Theory", hep-th/0010021.

[23] A. Sen, Int.J.Mod.Phys. A14 (1999) 4061.

[24] A. Sen, "Some issues in non-commutative tachyon condensation", Report No. MRI-P-000902, hepth/0009038. 